\title{
Construction Technology of Knowledge Graph and its Application in Power Grid
}

\author{
GAI Xiaoping ${ }^{1}$, RUAN Mengyu ${ }^{2 *}$, ZHANG Hong ${ }^{2}$, WU Ping ${ }^{3}$, REN Ruijun ${ }^{3}$ and GAO Feng ${ }^{2}$ \\ ${ }^{1}$ State Grid Gansu Electric Power Company, Lanzhou 730030, Gansu Province, China. \\ ${ }^{2}$ Beijing Kedong Electric Power Control System Co., Haidian District, Beijing 100192, China. \\ ${ }^{3}$ Training Centre of State Grid Gansu Electric Power Company, Lanzhou 730070, Gansu Province, China.
}

\begin{abstract}
With the rapid development of energy internet, dispatchers need to learn more knowledge with wider scope and fast update speed. It's urgent to realize the knowledge electrification, knowledge systematization, knowledge visualization and knowledge sharing of power grid dispatching. Firstly, this paper studies the definition and traits of knowledge graph, deeply analyses the key technologies of knowledge graph construction. Secondly, considering the contents and requirements of power grid dispatching business as well as the internal relationship of basic theoretical knowledge of power grid operation analysis, a method of constructing AC/DC power grid dispatching knowledge property graph is proposed. Finally, a practical example of a regional power grid knowledge graph is built to verify the effectiveness and practicality of the proposed method.
\end{abstract}

\section{Introduction}

Knowledge graph is the representative technology of knowledge engineering in the era of big data, the product of the combination of symbolism and connectionism, and the necessary foundation for artificial intelligence technology moving towards cognitive intelligence. In 2017, the State Council clearly points out in Development Plans of the New Generation Artificial Intelligence that knowledge graph is an important part of establishing the key generic technology system of new generation artificial intelligence [1], and a large amount of funds will be invested in the research on the theory, technology and application of knowledge graph. Since the concept of knowledge graph was put forward for 8 years, it has been successfully applied in the field of financial securities, library information, public security information analysis, stock investment information analysis, judicial assistant judgment, biomedical, e-commerce, agriculture, media, oil and gas, education and etc. [2], which has achieved great value and obvious effect.

With the continuous integration of large-scale clean energy and the continuous construction of large capacity long distance transmission lines, the profound changes of system operation scale, technical characteristics, interconnection mode and functional form constantly pose new challenges to power grid dispatching [3]. The scientific disposal of power grid dispatching faults and incidents requires dispatchers to deeply understand and master more and more knowledge, such as the deterministic knowledge of dispatching regulations and stability regulations, the operation characteristics of power grid, the principle of power grid fault disposal. Moreover, the knowledge updating speed is faster, and more factors need to be taken into account in dispatching decision-making, such as control requirements, power market trading plan, and new energy consumption under $\mathrm{AC} / \mathrm{DC}$ coupling operation mode. Thus, it is urgent to realize the digitalization, systematization and visualization of the knowledge in the field of power grid dispatching. At the same time, due to the fact that power grid dispatch and control relies on the accumulation of practical experience to a certain extent, it is also extremely urgent to realize domain knowledge sharing.

At present, the research on the construction of knowledge graph in the field of power grid is still at the exploratory stage, mainly focusing on the fields of power grid operation service [4-6], power grid equipment maintenance and inspection [7-11] as well as power grid dispatching [12-20]. For example, [15] constructs the dispatching automation platform and the business logic knowledge graph, which clearly shows the whole business logic and is convenient for auxiliary analysis. [16] proposes a model of power system operation knowledge for decision-making in dispatch with artificial intelligence, which provides real-time decision information for section control. [19] puts forward a framework of knowledgegraph-based fault handling system, which not only provides an important reference for the construction of knowledge graph in dispatch, but builds a solid foundation for the realization of intelligent control of power grid.

It is suggested that knowledge graph's concept and knowledge graph construction technology are studied. On the basis of fully understanding the knowledge characteristics of power grid dispatching, the source of

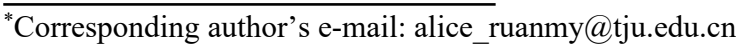


knowledge in dispatch is determined, and a method of constructing knowledge property graph model in $\mathrm{AC} / \mathrm{DC}$ power grid dispatching is proposed. It is expected to provide effective support for the automation and intelligence of dispatching business such as knowledge learning, skill training and online auxiliary decisionmaking.

\section{Definition and Construction Technology of Knowledge Graph}

\subsection{Definition of Knowledge Graph}

Knowledge Graph is a new stage in the development of knowledge engineering, and there are different opinions on its definition. The definition commonly used by far in china is proposed by the Language and Knowledge Computing Committee of the Chinese Information Society of China in 2018. It's suggested that knowledge graph is to describe the concepts, entities and their relationships in the objective world in a structured form, and to express the information of the Internet in a form closer to the human cognition of the world, providing a better ability to organize, manage and understand the massive amount of information on the Internet [21].

Compared with the previous stage of knowledge engineering development such as World Wide Web 1.0

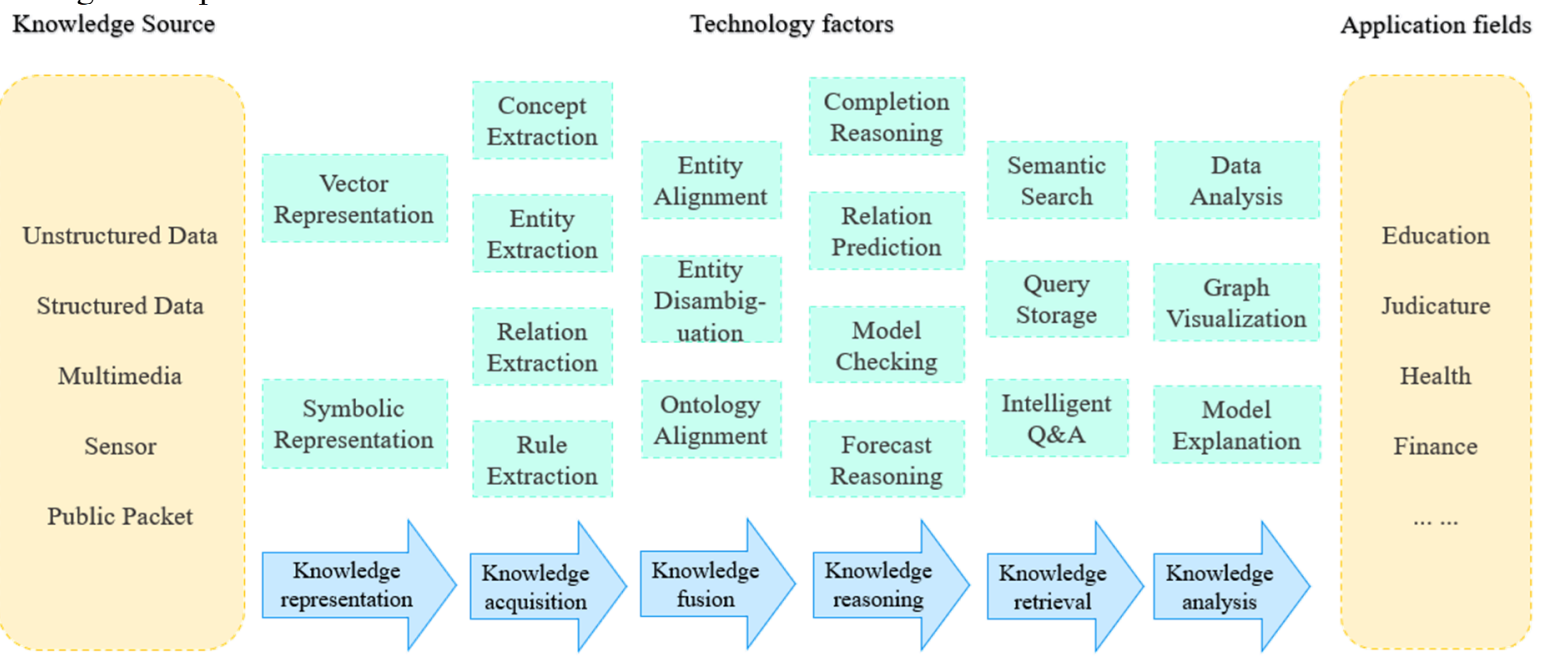

Fig. 1 Technology Process of Knowledge Graph ${ }^{[23]}$

There are many sources for knowledge data acquisition, such as unstructured data, semi-structured data, structured data. Knowledge data from different sources and structures need to be converted into elements that can be recognized and processed by computers, forming the data structure by knowledge representation technology [22]. In general, symbol-based knowledge representation methods (such as production rules, semantic web, frame method) and representation learningbased knowledge representation methods (such as vectorbased distributed representation method) are normally used. Then it is necessary to use different knowledge technologies like knowledge extraction and knowledge fusion in combination to automatically extract information from massive and complex unstructured data. and Swarm Intelligence Web2.0, the knowledge graph can obtain knowledge in the open Internet with big data, and use this massive knowledge to build a knowledge network providing smart services for all walks of life. At the same time, extensive interconnection promotes the knowledge accumulation, forming an iterative mutual enhancement process, which can realize the leap from Internet information services to intelligent knowledge services [22].

\subsection{Construction Technology of Knowledge Graph}

The general process of constructing a knowledge graph is shown in Figure 1. Firstly, determine the knowledge representation model and the knowledge description framework. Secondly, select appropriate knowledge acquisition methods according to different data sources. Then comprehensively use knowledge fusion, knowledge mining, and knowledge reasoning technology to do knowledge processing and quality assessment, further updating and improving the knowledge graph. Finally, according to the specific application requirements, design appropriate knowledge access and presentation methods [23]. Among them, the key technologies involved include: knowledge source, knowledge representation, knowledge acquisition, knowledge modelling, and knowledge storage. 
Knowledge modelling is the most important part in the construction of knowledge graphs, that is, to determine an ontology model to organize knowledge. Generally, there are two ways of it, top-down and bottom-up approaches [22]. At present, there are three types of knowledge graph models recognized in the industry, namely Resource Description Framework (RDF), Property Grapy and Hypergraph. Among them, RDF model and Property Graph model have been widely used. Compared with the RDF model composed of triples, the property graph model is composed of four basic data structures: nodes, relationships, properties, and labels where various graph algorithms are built on. That is to say, the property graph model makes full use of the graph nature of the data, so as to realize efficient knowledge reasoning and complex deep traversal. Therefore, this paper uses the property graph model to construct the knowledge graph of the power grid dispatching.

\section{Method for constructing knowledge graph of power grid dispatching}

\subsection{Determination of knowledge sources in the Field of Power Grid Dispatching}

According to the internal connection of the contents and requirements of power grid dispatching business and the basic theoretical knowledge of power grid operation analysis, the source of knowledge of the power grid dispatching is determined, including: standard subject word category, electric power industry standard category, power grid dispatching management regulations category, and necessary dynamic knowledge of power grid dispatching category. In other words, the standard subject word category refers to knowledges used to regulate the relationship between node names, species, and references, which are recorded in norms like Electric Power Thesaurus, Power Grid Dispatching Glossary. The electric power industry standard category refers to knowledges used to explain the basic concepts, calculation basis, and operation requirements of power calculation, which are recorded in norms like the Grid Operation Code, Guide on Security and Stability for Power System. The power grid dispatching management regulations category refers to knowledges used to explain the basic principles and procedures of abnormal disposal and incident handling, which are recorded in norms like Power Grid Dispatching Management Regulations and Implementation, State Grid Dispatch Control Management Regulation. The necessary dynamic knowledge of power grid dispatching category refers to knowledges on the main network stability regulations, accident disposal plan and operation mode of each actual power grid. Such knowledge is needed to be mastered by dispatchers and is constantly updated. For example, the main network stability regulations need to be formulated at least once a year.

\subsection{Construction of Knowledge Property Graph Model in the Field of Power Grid Dispatching}

Based on the declarative query language, a knowledge property graph model in the field of power dispatching is established, by extracting nodes, relationships, properties and labels from the knowledge source. In order to improve query efficiency, the properties of nodes and relationships should be as few as possible. That is to say, we need to establish thin nodes and relationships. According to the knowledge extraction scope and the knowledge purpose in dispatch, the basic principles of constructing the power gird dispatching knowledge property graph model are summarized as follows: (I) The knowledge of the definition type is expressed as properties. The definition does not need to be calculated, and can be used as properties of the end point, making the model simpler. (II) The source of knowledge is expressed as the properties of nodes or relationships. Storing the source of knowledge in the knowledge graph not only allows the dispatcher to clearly understand where the knowledge is from improving the credibility of the knowledge, but also can easily search all the knowledge of a certain source making the map of books. (III) Avoid the establishment of super nodes. The so-called super node means that some parts of the graph are all connected to the same node. Since graph traversal will traverse all connections with the node to determine the next traversal path, the existence of super nodes will greatly increase the searching time. It is necessary to avoid the establishment of super nodes as much as possible. In the knowledge of power grid dispatching, labels should be used to indicate broad terms such as "a certain regional power grid" and "defined section".

\section{Results and Discussions}

Taking the common dispatching knowledge of a certain regional power grid as an example, $\mathrm{Neo} 4 \mathrm{j}$ is used to construct a knowledge graph to verify the validity and practicability of the method of constructing a knowledge property graph model in the field of power grid dispatching proposed in this paper.

\subsection{Examples of Knowledge Graph construction method}

Knowledge point 1: "Inter-system tie lines generally refer to transmission lines between provincial power grids or between regional power grids. A regional power grid is a grid formed by interconnecting power grids between several provinces. Source: Guide on Security and Stability for Power System (DL755-2001) ".

The property graph model of knowledge point 1 is as follows:

(sysllx: Tie_Line \{name: "Inter-System Tie Line", definition: "Inter-system tie line generally refers to transmission lines between provincial power grids or between large regional power grids.", Source: "Guide on Security and Stability for Power System (DL755 -2001)"\}) 
(dqdianwang: term \{name: "Regional Power Grid", definition: "a grid formed by interconnecting several provincial power grids.", Source: "Guide on Security and Stability for Power System (DL755-2001)"\})

(sysllx)-[:Related_Terms]->(dqdianwang)

Knowledge point 2: "E-Yu section: 1000kV Nanjing I line, 500kV Woxian I, II line, Xiaoshi I, II loop lines are composed of power transmission section, Hubei to Henan is the positive direction $(+) "$.

The property graph model of knowledge point 2 is as follows:

(eyudm: section nname: "E-Yu section", positive_direction: "Hubei send Henan" $\}$ )[:Section_Composition]->(nanjingIx: Line \{name: "Nan Jing I line" $\}$ )

(eyudm)-[:Section_Composition]->(woxianIx:Line \{name:"Wo Xian I Line"\})

(eyudm)-[:Section_Composition]->(woxianIIx:Line \{name:"Wo Xian II Line"\})

(eyudm)-[:Section_Composition]->(xiaoshiIx:Line \{name:"Xiao Shi I Line"\})

(eyudm)-[:Section_Composition]->(xiaoshiIIx:Line \{name:"Xiao Shi II Line"\})

Knowledge point 3: "The basic process of incident disposal when the power of the transmission section exceeds the stability limit. The first step: Increase the output of the generators in the receiving system, reduce the output of the generators the sending system, and pay attention to increasing the voltage. The second step: If necessary, change the grid operation mode, adjust the distribution of power flow. The third step: If necessary, limit the load of the receiving system. Source: State Grid Dispatching Control Management Regulations (2014) 12.5".

The property graph model of knowledge point 3 is as follows:

(sddmyx: Theoretical_Knowledge \{name: "Transmission section overload", source: "State Grid Dispatching Control Management Regulations (2014) $\left.\left.12.5^{\prime \prime}\right\}\right)$

(sddmyx)-[:Disposal_Steps]->(dyb:Step\{name:" Step I", definition: "Increase the output of the generators in the receiving system, reduce the output of the generators the sending system, and pay attention to increasing the voltage.", Source: "State Grid Dispatching Control Management Regulations (2014) 12.5"\})

(dyb)-[:Next]->(deb:Step \{name:"Step II", definition: "If necessary, change the grid operation mode and adjust the power flow distribution.", Source: "State Grid Dispatching Control Management Regulations (2014) $\left.\left.12.5^{\prime \prime}\right\}\right)$

(deb)-[:Next]->(dsb:Step \{name: "Step III", definition: "If necessary, limit the load of the receiving system.", Source: "State Grid Dispatching Control Management Regulations (2014) 12.5"\}).

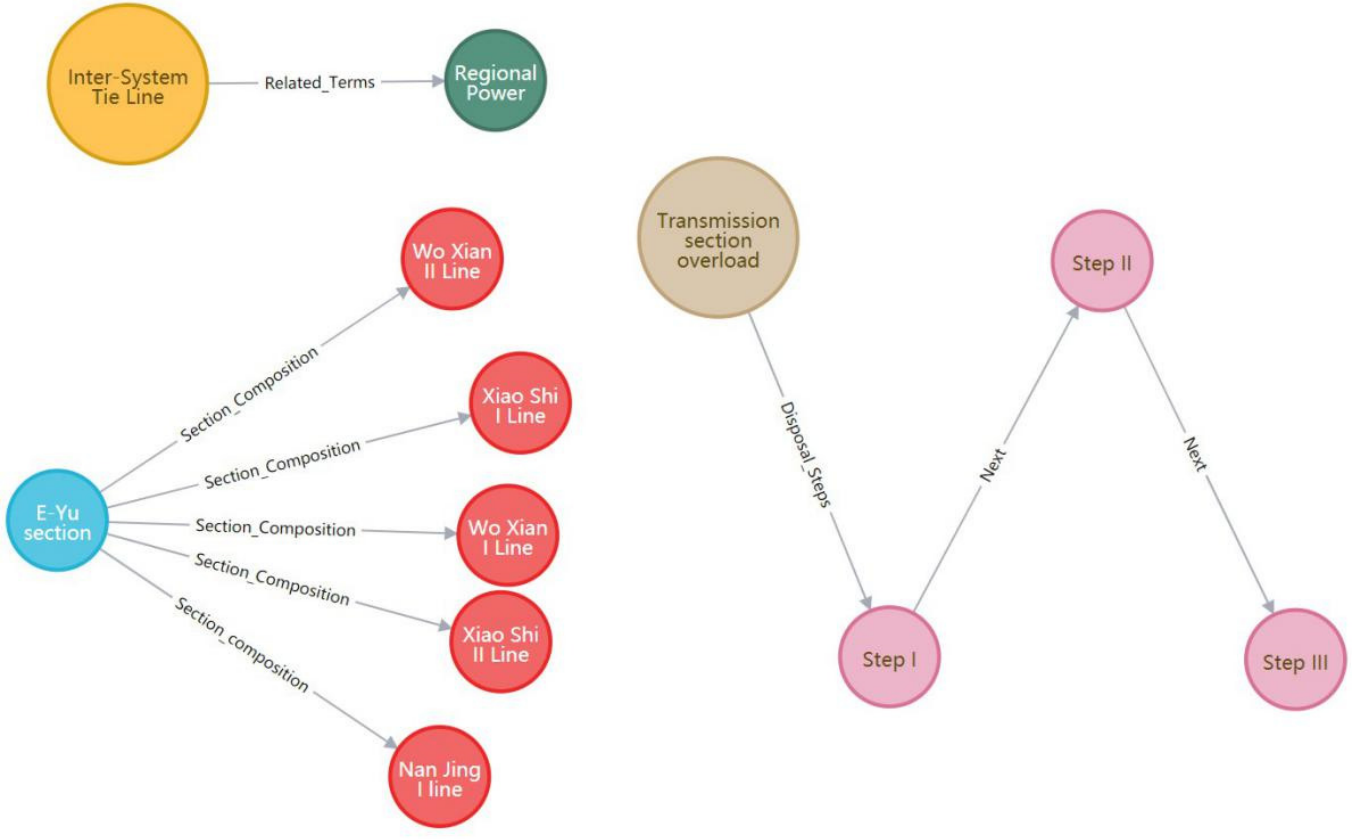

Fig. 2 Knowledge Graph of Knowledge 1/2/3

\subsection{Verification of Knowledge Graph Construction Principles}

Taking searching all the defined sections in a certain area as an example, this knowledge graph has a total of 530 nodes and 656 relationships. When all the defined sections in the area are connected to the same node "defined section", the node is a super node. When the principle (3) in section 3.2 is adopted to remove the super node and the "defined section" is used as the label of each section, the computation time for searching the names of all sections using two graph models is shown in Figure 3. It can be seen that in this example, the searching time of the super node graph is $135 \mathrm{~ms}$, and the search time of the property graph constructed by principle (3) is $38 \mathrm{~ms}$, which greatly reduces the time of knowledge query and knowledge reasoning. This proves the validity of the construction principle and the practicality of this construction method in power grids. 


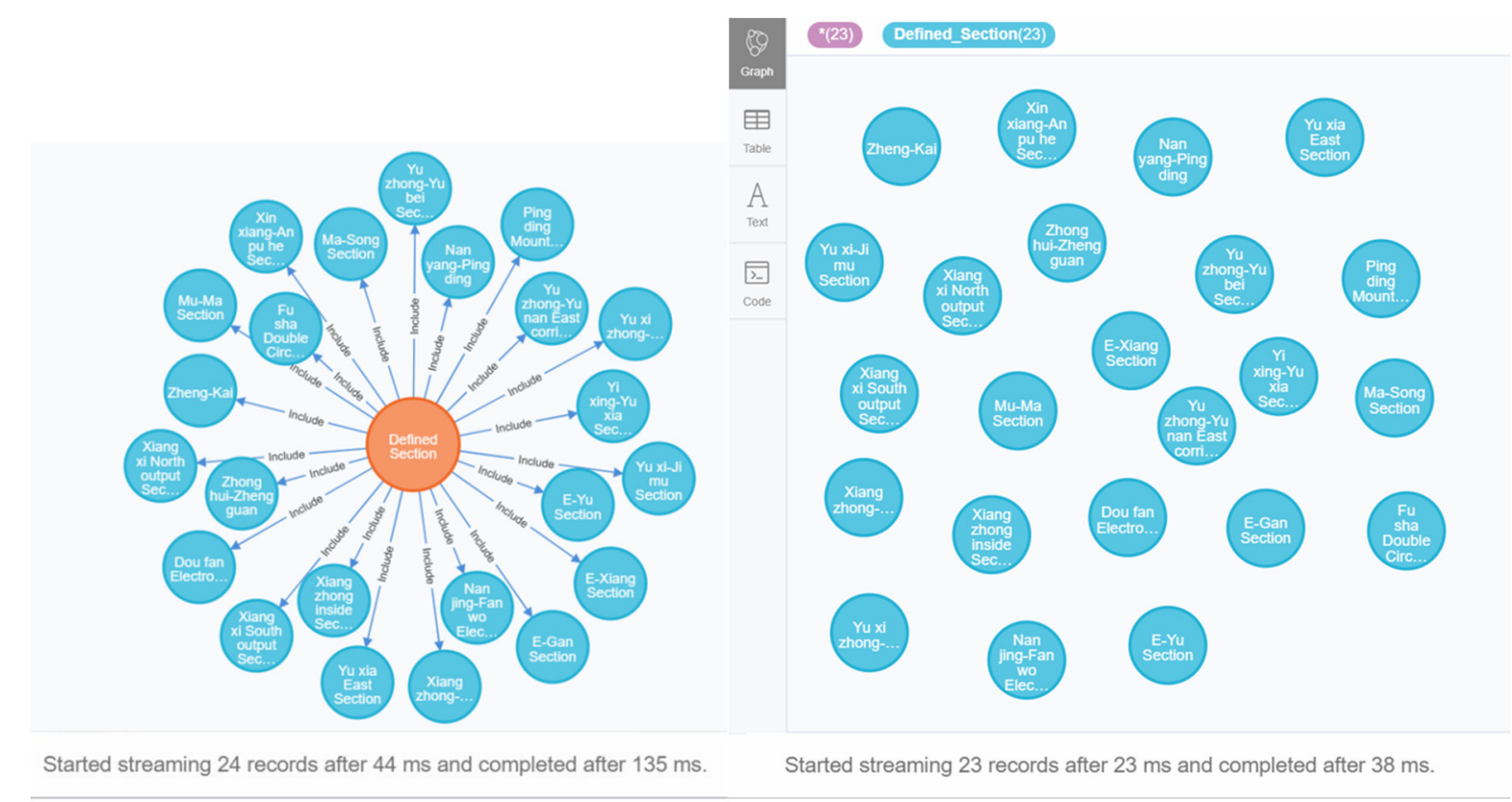

(a) Searching Time for Normal Knowledge Graph

(b) Searching Time for Our Property Graph

Fig. 3 Computation time comparison of two knowledge graph models

\section{Conclusions}

This paper first studies the key construction technologies and characteristics of the knowledge graph. Then based on the deep study of the power grid dispatching business knowledge and the basic theory of the power grid operation analysis, the knowledge source of power grid dispatching knowledge graph is determined, and the scope of knowledge extraction that needs to be covered in dispatch is also clarified. Finally, the basic principles of building property graph models are developed, and put into an actual power grid in a certain area of china to verify the applicability of the proposed knowledge graph construction method.

In the future, with the continuous development of related basic technologies such as knowledge inference engines, natural language processing, and distributed vector representation, knowledge graphs will be widely used in power grid. This trend will push traditional modeldriven dispatching control technology upgrading to data hybrid model-driven technology. Considering the needs of operation and management under the new situation, knowledge graph technology is going to be a strong support for the promotion of automatic and intelligent functions such as ubiquitous sensing of power grid state and adaptive cruise of power grid steady state.

\section{Acknowledgments}

This work is supported by State Grid Corporation Headquarters Technology Project (Research and application of knowledge service and skill training system for substation operation and maintenance 52467M200030).

\section{References}

1. The State Council. Development Plans of the New Generation Artificial Intelligence (GF [2017] No.35) [R]. Beijing: The Chinese Government Website, 2017. http://www.gov.cn/ zhengce/ zhengceku/201707/20/content 5211996.htm

2. WANG H.F, DING J, HU F.H, Wang X. Survey on Large Scale Enterprise-level Knowledge Graph Practices[J]. Computer Engineering, 2020,46(07):113.

3. LI M.J, TAO H.Z, XU H.Q, LIU J.B, ZHANG Q, ZHANG W. The Technical Framework and Application Prospect of Artificial Intelligence Application in the Field of Power Grid dispatching and Control[J]. Power System Technology, 2020, 44(02): 393-400.

4. TIAN X, LIU Y.H, WANG J, MENG W, LIN X.L. Rural Electrical. Application Value of Building Knowledge Graph System for Hotline Customer Service in State Grid Corporation[J]. Shandong Dianli Jishu[J], 2015,42(12):65-67+80.

5. LI Z.X, WAN L, ZHOU H.Y, WANG G.J, DENG L. Construction and Application of Operation and Business Target Knowledge Atlas Suited to Power Network Enterprise[J]. Rural Electrification, 2020(02):43-46.

6. WANG Y, PENG C.H, WANG Z.Q, FAN Q, YAO Y.Y, HUA Z.Y. Application of Knowledge Graph in Full-Service Unified Data Center of National Grid[J]. Computer Engineering and Applications, 2019,55(15):104-109.

7. PU T.J, QIAO J, HAN X, ZHANG G.B, WANG X.Y. Research and Application of Artificial Intelligence in 
Operation and Maintenance for Power Equipment[J]. High Voltage Engineering, 2020,46(02):369-383.

8. ZHAO Z.B, DUAN J.K, KONG Y.H, ZHANG D.X. Construction and Application of Bolt and Nut Pair Knowledge Graph Based on GGNN[J/OL]. Power System Technology:1-11. https://doi.org/10.13335/j.1000-3673.pst.2020.0063.

9. LIU Z.Q, WANG H.F. Retrieval Method for Defect Records of Power Equipment Based on Knowledge Graph Technology[J]. Automation of Electric Power System, 2018,42(14):158-164.

10. TANG Y.C, FANG D.J, HAN H.J, JIA R, ZHANG H.M, LIU T.T, LIU G.Y. Research on Power Equipment Quality Integrated Management System Based on Graph Database and Knowledge Graph[J]. Distribution \& Utilization, 2019,36(11):35-40.

11. LIU G.Y, HE M, ZHOU J.Q, TAN J, KAN B.W, FAN H, WEI L.F, DAI R.C. Active Distribution Network Integrated Energy Service Technical Support System Based on Graph Computing Platform[J]. Distribution \& Utilization, 2019,36(11): $3-11+54$.

12. SHAN X, LU X, ZHAI M.Z, et al. Analysis of Key Technologies for Artificial Intelligence Applied to Power Grid Dispatch and Control[J]. Automation of Electric Power Systems, 2019, 43(01): 49-57

13. FAN S.X, LI L.X, WANG S.Y, LIU X.W, YU Y.J, HAO B.W. Application Analysis and Exploration of Artificial Intelligence Technology in Power Grid Dispatch and Control[J]. Power System Technology, 2020, 44(02): 393-400.

14. GAO H.X, MIAO L, LIU J.N, DONG K, HE X.Z. Review on Knowledge Graph and Its Application in Power Systems[J]. Guangdong Electric Power,2020,33(09):66-76.

15. LI X.P, XU J.H, GUO Z.M, LI J.L, NING W.Y, WANG Z.X. Construction and Application of Knowledge Graph of Power Dispatching Automation System[J]. Electric Power, 2019,52(02):70-77+157.

16. SUN H.B, HUANG T.E, GUO Q.L, ZHANG B.M, GUO W.X, LIU W.T, XU T.S, XU T. Automatic Operator for Decision-making in Dispatch: Research and Applications[J]. Power System Technology, 2020,44(01):1-8.

17. ZHOU F, YE J.H, XIAO L.P, LIU H.Y, LOU T.Y. Research on Intelligent Question Answering System of power grid model ontology based on Knowledge graph[J]. China Science and Technology Information

18. LIU J, DU N, XU J, LIU X.Y, SONG Y.L, QIU L.P, ZHAO Y.G, SUN M.Y. Application and Research of Knowledge Graph in Electric Power Field[J]. Electric Power ICT, 2020,18(01):60-66.

19. QIAO J, WANG X.Y, MIN R, BAI S.H, YAO D, PU T.J. Framework and Key Technologies of Knowledge-graph-based Fault Handling System[J]. Proceedings of the CSEE, 2020,40(18):5837-5849.

20. YU J.M, WANG X.H, ZHANG Y, LIU Y, ZHAO S.G, SHAN L.F. Construction and application of knowledge graph for intelligent dispatching and control[J]. Power System Protection and Control,2020,48(03):29-35.

21. Knowledge Graph Development Report (2018) [R]. Special Committee on language and Knowledge Computing, Chinese information Processing society of China, 2018

22. White Book of Knowledge Graph Standardization (2019) [R]. China Electronics Standardization Institute, 2019.

23. WANG Q, MAO Z.D, WANG B, GUO L. Knowledge graph embedding: A survey of approaches and applications, IEEE Transactions on Knowledge \& Data Engineering, 2017, 29(12): 27242743. 